\title{
0 cuidado com os pés e a prevenção da úlcera em pacientes diabéticos no Brasil
}

\section{The care of feet and the prevention of ulcers in diabetic patients in Brazil}

Fábia Cheyenne Gomes de Morais Fernandes ${ }^{1}$ (D) Emelynne Gabrielly de Oliveira Santos ${ }^{1}$ (D), Juliana Ferreira Gomes de Morais ${ }^{1}$ (D), Lis Monique da Fonseca Medeiros ${ }^{1}$ (D), Isabelle Ribeiro Barbosa ${ }^{1}$ (D)

'Faculdade de Ciências da Saúde do Trairi, Universidade Federal do Rio Grande do Norte (UFRN) - Santa Cruz (RN), Brasil.

Como citar: Fernandes FCGM, Santos EGO, Morais JFG, Medeiros LMS, Barbosa IR. O cuidado com os pés e a prevenção da úlcera em pacientes diabéticos no Brasil. Cad Saúde Colet, 2020;28(2):302-310. https://doi.org/10.1590/1414$462 \times 202028020258$

\section{Resumo}

Introdução: O Diabetes Mellitus configura-se como um problema de saúde pública em ascensão e, uma vez mal controlado, favorece o desenvolvimento de complicações altamente incapacitantes, como o pé diabético. Objetivo: avaliar a prevalência e os fatores associados às ações de prevenção das úlceras dos pés em pacientes diabéticos no Brasil. Método: Trata-se de um estudo transversal que utilizou dados da Pesquisa Nacional de Saúde 2013, relativos à proporção de indivíduos com diagnóstico de diabetes em relação às recomendações do cuidado e a prevalência de úlcera nos pés. A PNS sorteou um total de 81.187 domicílios, sendo selecionado um indivíduo por domicílio, sendo entrevistadas 60.202 pessoas. Resultados: A realização do exame dos pés em portadores de diabetes por profissionais de saúde foi maior na Região Sudeste e a menor na Região Centro-Oeste. A não realização do exame foi mais prevalente no sexo feminino, em residente em área rural, de cor/raça parda, solteiro, sem instrução ou ensino fundamental incompleto. No Brasil, apenas 58,4\% dos pacientes diabéticos que fizeram consulta regular receberam a orientação para examinar os pés; a ocorrência de ferida no pé foi significativamente maior para o sexo masculino; na raça-cor preta/parda; a presença de ferida no pé foi significativamente maior entre os pacientes diabéticos que faziam consulta regularmente. Conclusão: Desse modo, no Brasil, a adesão dos profissionais na orientação para prevenção de úlceras diabéticas é considerada precária, com as menores prevalências de realização do cuidado nos grupos mais vulneráveis.

Palavras-chave: diabetes mellitus; complicações do diabetes; pé diabético; cuidado periódico; promoção da saúde.

\begin{abstract}
Background: Diabetes mellitus is a public health issue and once corrected, it favors the development of highly disabling complications, such as diabetic foot. Objective: This study aims to evaluate the prevalence and factors associated with actions to prevent foot ulcers in diabetic patients in Brazil. Method: This is a cross-sectional study using data from the National Health Survey 2013, regarding the diagnosis of diabetes related to the recommendations for care and a prevalence of foot ulcers. Results: The examination of patients with diabetes by health professionals was higher in the Southeast and Minor regions of the Central-West Region. Non-predominance of the test was more prevalent among females, in rural, color/race, single, uninstructed or incomplete elementary school subjects. In Brazil, only $58.4 \%$ of diabetic patients receiving regular consultation received guidance to examine the feet. The occurrence of sores was important for males; in black people/pardo Brazilians. The presence of foot wound was
\end{abstract}

Trabalho realizado na Universidade Federal do Rio Grande do Norte (UFRN) - Santa Cruz (RN), Brasil.

Correspondência: Emelynne Gabrielly de Oliveira Santos, Natal, RN, Brasil. E-mail: nellynha_15@hotmail.com

Fonte de financiamento: Coordenação de Aperfeiçoamento de Pessoal de Nível Superior.

Conflito de interesses: nada a declarar.

Recebido em: Jul. 07, 2018. Aceito em: Jul. 08, 2019
ISSN 2358-291X (Online) 
more significant among diabetic patients who had a regular visit. Conclusion: Thus, in Brazil, one of the guidelines for the prevention of diabetic ulcers is precarious, with lower prevalence of care in the most vulnerable groups.

Keywords: diabetes mellitus; diabetes complications; diabetic foot; episode of care; health promotion.

\section{INTRODUÇÃO}

O Diabetes Mellitus (DM) configura-se como um problema de saúde pública em ascensão, apresentando elevada morbimortalidade e alto índice de complicações que geram consequências de cunho econômico, social e psicológico, além da diminuição da qualidade de vida dos doentes e seus familiares'.

Mundialmente, o DM é uma doença que afeta 425 milhões de adultos, com mais de $80 \%$ das mortes por esta causa ocorrendo em países de baixa e média renda ${ }^{2}$. Segundo a Organização Mundial da Saúde (OMS), em 2012 ocorreu 1,5 milhão de mortes por esse agravo em todo o mundo, e a prevalência global de diabetes praticamente dobrou desde 1980, passando de $4,7 \%$ a $8,5 \%$ na população adulta ${ }^{3}$.

Dentre as complicações microvasculares, neuropáticas e macrovasculares acarretadas pelo DM, destacam-se as doenças vasculares periféricas e coronarianas. Além disso, o diabetes mal controlado favorece o desenvolvimento de complicações altamente incapacitantes, a exemplo do pé diabético, acarretando perdas de suas atividades diárias e laborais e elevados custos hospitalares para o Sistema de Saúde ${ }^{4,5}$.

Pé diabético é o termo designado para nomear as diversas alterações e complicações ocorridas, isoladamente ou em conjunto, nos pés e nos membros inferiores dos diabéticos, cujo resultado dessa complicação é um elevado custo humano e financeiro. As ações de prevenção dessa morbidade dependem de um bom controle da doença e da implantação de medidas relativamente simples de assistência preventiva, de diagnóstico precoce e de tratamento mais resolutivo nos estágios iniciais da doença. Contudo, o pé diabético em estágio terminal, necrosado e infectado é uma condição frequente em todos os serviços de emergência, resultado de ações precárias de prevenção e de meses ou anos de assistência inespecífica ${ }^{6}$.

Além de ser uma medida preventiva, o exame clínico dos pés é o método diagnóstico mais efetivo, simples e de baixo custo para identificação da neuropatia diabética. Apesar de muitos fatores de risco para ulceração/amputação poderem ser descobertos com o exame cuidadoso dos pés, a análise da história clínica e o exame dos pés não se constituem uma rotina nos serviços de saúde ${ }^{7}$.

O autoexame diário dos pés é outra medida de prevenção primária, uma vez que propicia a identificação precoce e tratamento oportuno das alterações encontradas. Estudos têm demonstrado que programas educacionais abrangentes, que incluem exame regular dos pés, classificação de risco e educação terapêutica, podem reduzir a ocorrência de lesões nos pés em até $50 \%^{8}$.

No âmbito nacional, está proposta a organização de uma rede de serviços para o cuidado dos pés de pessoas com DM, considerando o acompanhamento na atenção básica como ideal, por ser o nível de atenção mais próximo da população e responsável pelo cuidado longitudinal, integral e coordenado de sua população de referência. Assim, enfatiza a avaliação dos pés, com estratificação de risco e estabelecimento da periodicidade de acompanhamento, e a orientação para o autoexame dos pés como compromisso da Equipe de Saúde da Família no cuidado ao portador de diabetes ${ }^{9}$.

Nesse sentido, o reconhecimento dos fatores associados às ações de prevenção das úlceras dos pés em pacientes diabéticos podem direcionar mudanças em práticas assistenciais nos serviços de saúde dos diferentes níveis de atenção, bem como (re)pensar uma assistência mais efetiva, a partir da identificação de indivíduos e regiões mais vulneráveis, a fim de reduzir possíveis complicações.

O objetivo deste estudo é avaliar a prevalência e os fatores associados às ações de prevenção das úlceras dos pés em pacientes diabéticos no Brasil a partir dos dados da Pesquisa Nacional de Saúde de 2013. 


\section{MÉTODO}

Estudo transversal descritivo com dados provenientes da Pesquisa Nacional de Saúde (PNS) de 2013. A população-alvo do inquérito foi constituída por adultos ( $\geq 18$ anos de idade), residentes em domicílios particulares do território nacional. Não foram incluídos os setores censitários especiais (quartéis, bases militares, alojamentos, acampamentos, embarcações, penitenciárias, colônias penais, presídios, cadeias, asilos, orfanatos, conventos e hospitais).

A PNS sorteou um total de 81.187 domicílios, sendo selecionado um indivíduo por domicílio. Após o encerramento da coleta, foram realizadas entrevistas em 64.348 domicílios, e 60.202 com o morador selecionado, o que resultou em uma taxa de não resposta de $8,1 \%$. Desses, $47,1 \%$ corresponderam ao sexo masculino e $52,9 \%$ do sexo feminino; e a idade dos indivíduos variou de 18 a 101 anos, com valor da média igual a 43 e mediana de 41 . 0 plano amostral empregado foi o de amostragem por conglomerados em três estágios, com estratificação das unidades primárias de amostragem. Os setores censitários ou conjunto de setores formam as unidades primárias de amostragem (UPA), os domicílios representam as unidades de segundo estágio, e os moradores adultos definem as unidades de terceiro estágio.

O questionário da PNS foi divido em módulos, que contemplam características do domicílio, de todos os moradores e do morador adulto selecionado. Através do Módulo $\mathrm{Q}$, a PNS investigou as Doenças Crônicas, especificamente a Diabetes autorreferida entre indivíduos de 18 anos. Para efeito da presente pesquisa, foram considerados diabéticos os indivíduos que responderam positivamente ao questionamento: "Algum médico já Ihe deu o diagnóstico de diabetes?".

A partir desta variável, foram analisadas: "Proporção (\%) de pessoas com diagnóstico de diabetes que tiveram seus pés examinados nos últimos 12 meses anteriores à pesquisa"; "Proporção (\%) de pessoas com diagnóstico de diabetes que receberam a recomendação de examinar os pés regularmente" $e$ "Proporção (\%) de pessoas com diagnóstico de diabetes que tiveram úlcera ou ferida nos pés".

As três variáveis foram analisadas de acordo com os seguintes aspectos sociodemográficos: as macrorregiões (Norte, Nordeste, Sul, Sudeste e Centro-Oeste), situação do domicílio (urbano/rural), cor da pele, sexo, estado civil, nível de instrução, frequência de realização de consulta, último exame dos pés realizado, relato de úlceras nos pés e o uso de medicação oral para DM e insulina.

O presente estudo avaliou os dados da PNS que estavam disponíveis no site do Datasus ${ }^{10}$, e as análises foram realizadas entre dezembro de 2016 e janeiro de 2017. Os dados foram apresentados a partir de valores de proporções, média e intervalo de confiança a 95\%. A análise da significância entre os dados foi avaliada a partir da intercessão dos intervalos de confiança. Como foram utilizados somente os dados disponibilizados pelo Datasus, os pesquisadores só tiveram acesso aos valores relativos de cada evento, não tendo acesso aos valores absolutos.

O presente estudo utiliza dados secundários da PNS disponíveis em sites oficiais do Ministério da Saúde do Brasil, sendo dispensado de apreciação em comitê de ética em pesquisa, em conformidade com a Resolução 466/2012 do Conselho Nacional de Saúde.

\section{RESULTADOS}

A realização do exame dos pés em portadores de diabetes por profissionais de saúde, nos últimos 12 meses, obteve maior percentual na Região Sudeste $\left(38,6 \%\right.$; IC $\left.{ }_{95 \%} 33,9-43,3\right)$ e o menor na Região Centro-Oeste $\left(21,4 \% ; C_{95 \%} 16,3-26,5\right)$ (Figura 1).

Aos diabéticos que nunca tiveram os pés examinados, foi significativamente maior para o sexo feminino $\left(58,6 \%\right.$; IC $\left.{ }_{95 \%} 55,06-62,32\right)$, residente em área rural $\left(67,3 \%\right.$; IC $\left.C_{95 \%} 59,92-74,86\right)$, de cor/raça parda $\left(59,2 \% ; I_{95 \%} 54,80-63,61\right)$, solteiro $\left(57,5 \%\right.$; IC $\left.C_{95 \%} 51,34-63,67\right)$, sem instrução ou ensino fundamental incompleto $\left(58,3 \% ; \mathrm{IC} \mathrm{C}_{95 \%} 54,64-62,12\right)$, que não faz a consulta regular para diabetes $\left(66,96 \% \mathrm{IC}_{95 \%} 60,99-72,93\right)$ (Tabela 1$)$.

No Brasil, pouco mais da metade dos pacientes com diabetes receberam a recomendação para examinar os pés regularmente $\left(53,6 \% ; \mathrm{IC}_{95 \%} 50,79-56,55\right)$, com maior percentual para a Região Norte $\left(61,9 \%\right.$; IC $\left.{ }_{95 \%} 52,14-71,82\right)$ e menor para a Região Nordeste $(46,9 \%$; 


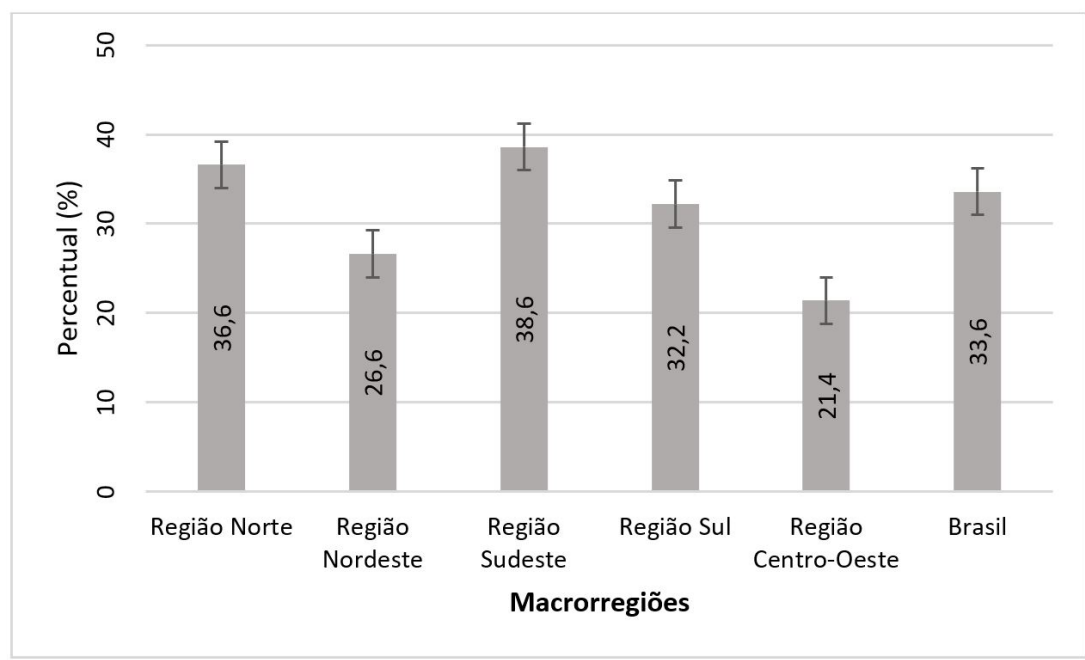

Figura 1. Prevalência dos pacientes diabéticos que tiveram os pés examinados por profissional de saúde, de acordo com as macrorregiões do Brasil. Pesquisa Nacional de Saúde, 2013

Tabela 1. Prevalência dos pacientes diabéticos maiores de 18 anos que nunca tiveram os pés examinados por profissional de saúde, de acordo com variáveis sociodemográficas e de saúde. Pesquisa Nacional de Saúde, 2013.

\begin{tabular}{|c|c|}
\hline VARIÁVEIS & Nunca teve seus pés examinados ( IC ${ }_{95 \%}^{*}$ ) \\
\hline \multicolumn{2}{|l|}{ SITUAÇÃO DOMICÍLIO } \\
\hline Urbano & $53,67(50,48-56,87)$ \\
\hline Rural & $67,39(59,92-74,86)$ \\
\hline \multicolumn{2}{|l|}{ SEXO } \\
\hline Masculino & $49,93(44,57-55,29)$ \\
\hline Feminino & $58,69(55,06-62,32)$ \\
\hline \multicolumn{2}{|l|}{ COR OU RAÇA } \\
\hline Branca & $52,68(48,31-57,05)$ \\
\hline Preta & $54,33(46,00-62,65)$ \\
\hline Parda & $59,2(54,80-63,61)$ \\
\hline \multicolumn{2}{|l|}{ ESTADO CIVIL } \\
\hline Casado(a) & $56,46(51,87-61,05)$ \\
\hline Separado(a) ou desquitado(a) judicialmente & $56,63(39,28-73,98)$ \\
\hline Divorciado(a) & $50,49(40,80-60,17)$ \\
\hline Viúvo(a) & $49,12(42,93-55,31)$ \\
\hline Solteiro(a) & $57,5(51,34-63,67)$ \\
\hline \multicolumn{2}{|l|}{ NÍVEL DE INSTRUÇÃO } \\
\hline Sem instrução e fundamental incompleto & $58,38(54,64-62,12)$ \\
\hline Fundamental completo e médio incompleto & $54,36(45,56-63,17)$ \\
\hline Médio completo e superior incompleto & $51,65(44,16-59,14)$ \\
\hline Superior completo & $40,36(29,65-51,07)$ \\
\hline \multicolumn{2}{|l|}{ FAZ CONSULTA REGULAR } \\
\hline Sim & $49,7(46,22-53,17)$ \\
\hline Não & $66,96(60,99-72,93)$ \\
\hline
\end{tabular}

*IC: Intervalo de Confiança

$\left.\mathrm{IC}_{95 \%} 41,66-52,26\right)$. Os percentuais de recomendação para as demais regiões foram: $\mathrm{Sul}(55,12 \%)$, Sudeste $(55,8 \%)$ e Centro-Oeste $(50,55 \%)$.

Apenas 58,4\% (IC $\left.{ }_{95 \%} 54,97-61,85\right)$ dos pacientes diabéticos que fizeram consulta regular receberam a orientação para examinar os pés. Percebe-se que, quanto menor a frequência de exames dos pés, menor a prevalência de recomendação de realizar o exame. Ter ferida no pé obteve percentual maior entre os que receberam a recomendação para examinar os pés regularmente $\left(70,81 \% \mathrm{IC}_{95 \%} 58,37-83,24\right)$ (Tabela 2$)$. 
Tabela 2. Prevalência dos pacientes diabéticos que receberam a recomendação de examinar os pés regularmente, de acordo com variáveis de acesso e condições de saúde. Pesquisa Nacional de Saúde, 2013.

\begin{tabular}{|c|c|c|c|c|}
\hline & \multicolumn{2}{|c|}{ Receberam recomendação } & \multicolumn{2}{|c|}{$\begin{array}{l}\text { Não receberam } \\
\text { recomendação }\end{array}$} \\
\hline & $\%$ & IC*95\% & $\%$ & IC*95\% \\
\hline \multicolumn{5}{|l|}{ FAZ CONSULTA REGULAR } \\
\hline Sim & 58,41 & $54,97-61,85$ & 41,59 & $38,15-45,03$ \\
\hline Não & 44,73 & $38,03-51,44$ & 55,27 & $48,56-61,97$ \\
\hline \multicolumn{5}{|l|}{ ÚLTIMO EXAME DOS PÉS } \\
\hline Há menos de 6 meses & 78,28 & $73,19-83,38$ & 21,72 & $16,62-26,81$ \\
\hline Entre 6 meses e menos de 1 ano & 78,6 & $69,84-87,36$ & 21,4 & $12,64-30,16$ \\
\hline Entre 1 ano e menos de 2 anos & 79,94 & $71,52-88,35$ & 20,06 & $11,65-28,48$ \\
\hline Entre 2 anos e menos de 3 anos & 41,38 & $22,99-59,78$ & 58,62 & $40,22-77,01$ \\
\hline 3 anos ou mais & 58,69 & $45,37-72,01$ & 41,31 & $27,99-54,63$ \\
\hline Nunca & 36,53 & $32,75-40,32$ & 63,47 & $59,68-67,25$ \\
\hline \multicolumn{5}{|l|}{ TEVE FERIDA NO PÉ } \\
\hline Sim & 70,81 & $58,37-83,24$ & 29,19 & $16,76-41,63$ \\
\hline Não & 52,56 & $49,65-55,46$ & 47,44 & $44,54-50,35$ \\
\hline
\end{tabular}

*IC: Intervalo de Confiança

Nos pacientes diabéticos, a ocorrência de ferida no pé foi significativamente maior para o sexo masculino $\left(7,25 \% ; \mathrm{IC}_{95 \%} 4,37-10,13\right)$; na raça-cor preta/parda $\left(6,72 \% ; \mathrm{IC}_{95 \%} 4,15-9,29\right)$; estado civil viúvo (7,1\%; IC95\% 3,82-10,38); que reside em área urbana (6,27\%; IC $\left.{ }_{95 \%} 4,57-7,98\right)$; com nível de instrução mais baixo ( $7,6 \%$ sem instrução e fundamental incompleto $\left.I C_{95 \%} 5,31-9,90\right)$ (Tabela 3).

Tabela 3. Prevalência dos pacientes diabéticos que tiveram úlcera ou ferida nos pés, de acordo com as variáveis sociodemográficas. Pesquisa Nacional de Saúde, 2013.

\begin{tabular}{|c|c|c|c|c|}
\hline & \multicolumn{2}{|c|}{ Presença de úlcera } & \multicolumn{2}{|c|}{ Ausência de úlcera } \\
\hline & $\%$ & IC*95\% & $\%$ & IC*95\% \\
\hline \multicolumn{5}{|l|}{ SEXO } \\
\hline Masculino & 7,25 & $4,37-10,13$ & 92,75 & $89,87-95,63$ \\
\hline Feminino & 5,3 & $3,57-7,03$ & 94,7 & $92,97-96,43$ \\
\hline \multicolumn{5}{|l|}{ COR OU RAÇA } \\
\hline Branca & 6,1 & $3,78-8,42$ & 93,9 & $91,58-96,22$ \\
\hline Preta/Parda & 6,72 & $4,15-9,29$ & 93,28 & $90,71-95,85$ \\
\hline \multicolumn{5}{|l|}{ DOMICÍLIO } \\
\hline Urbano & 6,27 & $4,57-7,98$ & 93,73 & $92,02-95,43$ \\
\hline Rural & * & * & 95,42 & $92,34-98,50$ \\
\hline \multicolumn{5}{|l|}{ ESTADO CIVIL } \\
\hline Casado & 5,26 & $3,30-7,23$ & 94,74 & $92,77-96,70$ \\
\hline Viúvo & 7,1 & $3,82-10,38$ & 92,9 & $89,62-96,18$ \\
\hline Solteiro & 7,63 & $0,65-12,62$ & 92,37 & $87,38-97,35$ \\
\hline \multicolumn{5}{|l|}{ NÍVEL DE INSTRUÇÃO } \\
\hline $\begin{array}{l}\text { Sem instrução e fundamental } \\
\text { incompleto }\end{array}$ & 7,6 & $5,31-9,90$ & 92,4 & $90,10-94,69$ \\
\hline $\begin{array}{l}\text { Fundamental completo e médio } \\
\text { incompleto }\end{array}$ & * & * & 95,07 & $92,37-97,77$ \\
\hline $\begin{array}{l}\text { Médio completo e superior } \\
\text { incompleto }\end{array}$ & * & * & 95,49 & $92,15-98,82$ \\
\hline Superior completo & * & * & 99,19 & $98,16-100,21$ \\
\hline
\end{tabular}

*Número de casos menor que 30, insuficiente para determinar qualquer estimativa com precisão aceitável; *IC: Intervalo de Confiança 
Foi possível constatar que a presença de ferida no pé foi significativamente maior entre os pacientes diabéticos que faziam consulta regularmente $\left(6,98 \% ; \mathrm{IC}_{95 \%} 4,99-8,97\right)$; que tiveram os pés examinados há menos de 6 meses $\left(9,99 \% ; I_{95 \%} 6,40-13,58\right)$; que utilizaram insulina nas últimas 2 semanas (14,71\%; IC $\left.C_{95 \%} 9,47-19,95\right)$; e que não usaram medicação oral para diabetes nas últimas 2 semanas $\left(7,84 \% ; I_{95 \%} 3,32-12,35\right)$ (Tabela 4$)$.

Tabela 4. Prevalência dos pacientes diabéticos que tiveram úlcera ou ferida nos pés, de acordo com variáveis de assistência à saúde. Pesquisa Nacional de Saúde, 2013.

\begin{tabular}{|c|c|c|c|c|}
\hline & \multicolumn{2}{|c|}{ Presença de úlcera } & \multicolumn{2}{|c|}{ Ausência de úlcera } \\
\hline & $\%$ & IC*95\% & $\%$ & IC*95\% \\
\hline \multicolumn{5}{|l|}{ FAZ CONSULTA REGULAR } \\
\hline Sim & 6,98 & $4,99-8,97$ & 93,02 & $91,03-95,01$ \\
\hline Não & 3,67 & $1,82-5,51$ & 96,33 & $94,49-98,18$ \\
\hline \multicolumn{5}{|l|}{ ÚLTIMO EXAME DOS PÉS } \\
\hline Há menos de 6 meses & 9,99 & $6,40-13,58$ & 90,01 & $86,42-93,60$ \\
\hline Entre 6 meses e 1 ano & * & * & 96,74 & $94,51-98,98$ \\
\hline Entre 1 ano e 2 anos & * & * & 97,38 & $94,73-100,02$ \\
\hline Entre 2 anos e 3 anos & * & * & 94,86 & $89,28-100,43$ \\
\hline 3 anos ou mais & * & * & 87,33 & $76,83-97,83$ \\
\hline Nunca & 4,37 & $2,40-6,33$ & 95,63 & $93,67-97,60$ \\
\hline \multicolumn{5}{|l|}{$\begin{array}{l}\text { USOU MEDICAÇÃO ORAL } \\
\text { PARA DM NAS ÚLTIMAS } \\
2 \text { SEMANAS }\end{array}$} \\
\hline Sim & 5,64 & $4,11-7,17$ & 94,36 & $92,83-95,89$ \\
\hline Não & 7,84 & $3,32-12,35$ & 92,16 & $87,65-96,68$ \\
\hline \multicolumn{5}{|l|}{$\begin{array}{l}\text { USOU INSULINA NAS } \\
\text { ÚLTIMAS } 2 \text { SEMANAS }\end{array}$} \\
\hline Sim & 14,71 & $9,47-19,95$ & 85,29 & $80,05-90,53$ \\
\hline Não & 4,04 & $2,61-5,46$ & 95,96 & $94,54-97,39$ \\
\hline
\end{tabular}

*Número de casos menor que 30, insuficiente para determinar qualquer estimativa com precisão aceitável; *IC: Intervalo de Confiança

\section{DISCUSSÃO}

O presente estudo observou que há uma baixa proporção de realização do exame dos pés em portadores de diabetes, com importantes desigualdades entre as regiões brasileiras. A carência da realização do exame e da recomendação para realizá-lo esteve associada às piores condições sociais e econômicas.

Outros autores também ressaltaram em suas pesquisas que o exame e as orientações referentes aos pés de pacientes diabéticos constituem práticas que ainda não foram incorporadas às ações cotidianas da Atenção Básica, implicando importante fator de risco ao desenvolvimento de ulcerações nos pés e suas complicações ${ }^{8,11}$.

Os dados deste estudo corroboram os achados de Bezerra et al. ${ }^{12}$, que investigaram pacientes diabéticos na atenção primária de Recife $(\mathrm{PE})$. Com relação à realização do exame dos pés pelos profissionais da equipe da Saúde da Família, 83,3\% dos usuários referiram não ter seus pés examinados durante as consultas, e 62,5\% relataram a não realização do autocuidado 
com os pés. Vale destacar que, dentre os usuários avaliados, 39,6\% apresentavam risco para desenvolver úlcera no pé.

No estudo sobre a prevalência do risco para desenvolver lesões entre 31 portadores de Diabetes Mellitus atendidos na Estratégia de Saúde da Família de Diamantina/MG, observou-se que apenas $32,3 \%$ tinham o hábito de avaliar os pés, $25,8 \%$ tinham o corte de unha inadequado e $71 \%$ usavam calçado inapropriado. Na avaliação dos pés, o estudo apontou que o risco para desenvolver complicações ulcerativas nos pés foi de 32,3\% de indivíduos com grau 2, e 6,5\% com grau 3; observaram-se ainda rachaduras (35,5\%), pele ressecada (29\%), unhas espessadas $(19,4 \%)$, hálux valgo (12,9\%), presença de calos $(6,5 \%)$ e sensibilidade diminuída $(6,5 \%)^{13}$.

A ausência da avaliação dos pés em portadores de diabetes, com a consequente carência de ações de educação para as práticas de cuidado com os pés, pressupõe o aumento do risco ao desenvolvimento de ulcerações principalmente para aqueles em condições sociais desfavoráveis. Atrelado a isso, tem-se a baixa escolaridade, que pode contribuir para aumentar a chance de complicações posteriores devido à limitação do acesso à informação e comprometimento da compreensão de orientações para o autocuidado ${ }^{4,13}$.

A educação e as práticas em saúde têm a finalidade de atingir o paciente, gerenciando a sua autonomia, para o exercício do autocuidado. Um exemplo de como essas ações podem ser efetivas na mudança de práticas foi a implantação de um programa de educação para enfermeiros em unidades de hemodiálise, sobre a necessidade de avaliações regulares dos pés de portadores de diabetes em diálise, o qual promoveu uma mudança na frequência com que os enfermeiros examinaram os pés dos pacientes e no comportamento do autocuidado com os pés, refletindo em maior conscientização sobre o risco nessa população'.

No estudo realizado em um hospital da rede pública de Pernambuco, verificou-se que a falta de orientação sobre o cuidado com os pés estava fortemente associada à ocorrência de amputações. Da mesma forma, a não realização do exame dos pés no último ano para prevenção ou controle do pé diabético mostrou uma probabilidade de 3,39 vezes de os pacientes serem submetidos a uma amputação. A falta de orientação quanto aos cuidados com os pés representou chance de 3,6 vezes de os pacientes necessitarem de amputação ${ }^{11}$.

Todavia, em um estudo realizado em Washington-EUA, apenas 29,5\% dos participantes relataram realizar o autoexame dos pés diariamente, apesar do alto nível de educação dos participantes (88\% apresentavam nível superior). Ao comparar os participantes que relataram realizar exames diários nos pés e aqueles que não realizavam exames diários nos pés, houve diferenças significativas entre as barreiras percebidas, como estar muito ocupado e perceber os exames dos pés como algo que não é importante ${ }^{14}$.

Semelhante aos dados do presente estudo sobre a ocorrência de lesão no pé, uma pesquisa realizada em Portugal caracterizou pacientes com diabetes tipo 2 com úlcera no pé, também obteve predominância do sexo masculino $(72,3 \%)$, com média de 4,55 anos da educação formal e maior prevalência entre os casados (63\%). O que se destaca nesse estudo é o elevado acesso dos pacientes ao serviço de saúde e aos cuidados específicos com os pés. Os pacientes tinham acesso aos cuidados de saúde regularmente, $65,5 \%$ foram atendidos em 4 consultas anuais no centro de saúde no ano anterior e $62 \%$ foram submetidos ao monitoramento em uma clínica de pé diabético ${ }^{15}$.

O estudo realizado com diabéticos em clínicas públicas na Tanzânia afirmou que pacientes que tinham pelo menos um exame de pé realizado por um profissional de saúde eram mais propensos a cuidar dos seus pés do que aqueles que não o tinham. Ainda se comprovou nesse estudo que $48 \%$ receberam conselhos sobre cuidados com os pés, e $27,5 \%$ tiveram os pés examinados pelo menos uma vez desde o diagnóstico inicial. A partir dos estudos descritos acima, percebe-se a grande discrepância com o observado no Brasil, onde se observa a baixa prevalência de realização de consultas regulares, e mesmo aqueles que o fazem, não recebem orientações adequadas ${ }^{16}$.

Assim, a importância deste estudo para a Saúde Coletiva reside no fato de permitir o reconhecimento dos fatores associados às ações de prevenção das úlceras em pacientes diabéticos no Brasil. A partir disso, é possível planejar estratégias de prevenção mais efetivas, bem como fortalecer ações de promoção à saúde, com foco na redução dessa morbidade, sobretudo em regiões mais vulneráveis. 
Algumas limitações devem ser observadas na apreciação dos resultados aqui apresentados, quanto à validade e à reprodutibilidade dos indicadores apresentados. Apesar deste estudo ser conduzido pela Pesquisa Nacional de Saúde, com comprovada validade metodológica, trata-se de informações autorreferidas pelos entrevistados, sendo possível ocorrer diferenças na compreensão dos entrevistados, viés de recordatório, sub ou superestimação dos valores referidos.

O exame físico dos pés e a orientação para o autoexame dos pés ainda têm baixa adesão por profissionais de saúde, implicando aumento da hospitalização e risco de complicações e amputações nos portadores de diabetes, com consequente prejuízos sociais e financeiros.

Além disso, o autoexame dos pés diariamente permite empoderar o paciente no seu autocuidado, melhorando sua qualidade de vida e autonomia sobre a doença, que associado à educação em saúde, seja individual ou em grupo, pode proporcionar mudança de hábitos e cuidado efetivo com sua saúde.

No âmbito nacional, ter ferida no pé foi mais prevalente entre diabéticos com possível déficit de autocuidado e com índices glicêmicos mais elevados que necessitaram do uso da insulina. Para os diabéticos que faziam consulta regularmente e que tiveram os pés examinados, o diagnóstico de ulceração ocorreu mais facilmente.

Desse modo, a úlcera no pé de portadores de diabetes é considerada prevenível, através de medidas de cuidados e conforto para os pés, atenção integral ao portador da diabetes com controle do índice glicêmico e ampliação da rede de atenção à saúde do portador de diabetes.

Logo, acredita-se que o fortalecimento da prática de cuidado com os pés e a busca ativa dos portadores de diabetes na atenção primária à saúde pode ser uma importante estratégia para melhoria do cenário nacional.

\section{REFERÊNCIAS}

1. Brand SL, Musgrove A, Jeffcoate WJ, Lincoln NB. Evaluation of the effect of nurse education on patient reported foot checks and foot care behavior of people with diabetes receiving haemodialysis. Diabet Med. 2016;33(2):204-7. http://dx.doi.org/10.1111/dme.12831. PMid:26042333.

2. International Diabetes Federation. IDF diabetes atlas. 8th ed. Brussels: IDF; 2017.

3. World Health Organization. Global report on diabetes. Geneva: WHO; 2016.

4. Ochoa-Vigo K, Torquato MTCG, Silvério IAS, Queiroz FA, De-La-Torre-Ugarte-Guanilo MC, Pace AE. Caracterização de pessoas com diabetes em unidades de atenção primária e secundária em relação a fatores desencadeantes do pé diabético. Acta Paul Enferm. 2006;19(3):296-303. http://dx.doi.org/10.1590/ S0103-21002006000300007.

5. Santos AL, Cecílio HP, Teston EF, Arruda GO, Peternella FM, Marcon SS. Complicações microvasculares em diabéticos Tipo 2 e fatores associados: inquérito telefônico de morbidade autorreferida. Cien Saude Colet. 2015;20(3):761-70. http://dx.doi.org/10.1590/1413-81232015203.12182014. PMid:25760116.

6. Caiafa JS, Castro AA, Fidelis C, Santos VP, Silva ES, Sitrângulo C Jr. Atenção integral ao portador de pé diabético. J Vasc Bras. 2011;10(4):1-32. http://dx.doi.org/10.1590/S1677-54492011000600001.

7. Sociedade Brasileira de Diabetes. Diretrizes da Sociedade Brasileira de Diabetes: 2015-2016 [Internet]. São Paulo: A.C. Farmacêutica; 2016. [citado em 2018 Jan 20]. 348 p. Disponível em: http://www.diabetes. org.br/profissionais/images/docs/DIRETRIZES-SBD-2015-2016.pdf

8. Cubas MR, Santos OM, Retzlaff EMA, Telma HLC, Andrade IPS, Moser ADL, et al. Pé diabético: orientações e conhecimento sobre cuidados preventivos. Fisioter Mov. 2013;26(3):647-55. http://dx.doi.org/10.1590/ S0103-51502013000300019.

9. Brasil. Ministério da Saúde. Manual do pé diabético. Estratégias para o cuidado da pessoa com doença crônica. Brasília: Ministério da Saúde; 2016.

10. Departamento de Informática do Sistema Único de Saúde [Internet]. Brasília: Ministério da Saúde; 2018 [citado em 2018 Jan 20]. Disponível em: www.datasus.gov.br

11. Santos ICRV, Carvalho EF, Souza WV, Albuquerque EC. Fatores associados a amputações por pé diabético. J Vasc Bras. 2015;14(1):37-45. http://dx.doi.org/10.1590/1677-5449.20140049.

12. Bezerra GC, Santos ICRV, Lima JC, Souza MAO. Avaliação do risco para desenvolver pé diabético na atenção básica [Internet]. Rev Estima. 2015;13(3). [citado em 2018 Jan 20]. Disponível em: https://www. revistaestima.com.br/index.php/estima/article/view/108 
13. Paula DB, Martins DA, Lara MO, Stuchi RAG, Lima AMJ, Azevedo DSS. Avaliação dos pés em indivíduos portadores de diabetes atendidos em uma unidade de atenção primária. Rev Enferm UFPE on line. 2016;10(6):4751-6.

14. Pocuis J, Li SM, Janci MM, Thompson HJ. Exploring diabetic foot exam performance in a specialty clinic. Clin Nurs Res. 2017;26(1):82-92. http://dx.doi.org/10.1177/1054773815596699. PMid:26204900.

15. Pedras S, Carvalho R, Pereira MG. Sociodemographic and clinical characteristics of patients with diabetic foot ulcer. Rev Assoc Med Bras. 2016;62(2):171-8. http://dx.doi.org/10.1590/1806-9282.62.02.171. PMid:27167548.

16. Chiwanga FS, Njelekela MA. Diabetic foot: prevalence, knowledge, and foot self-care practices among diabetic patients in Dar es Salaam, Tanzania - a cross-sectional study. J Foot Ankle Res. 2015;8(20):20. http://dx.doi.org/10.1186/s13047-015-0080-y. PMid:26064190. 